\title{
Primary Central Nervous System T-cell Lymphoma as Methotrexate-associated Lymphoproliferative Disorders: Case Report
}

\author{
Hiroto KaWANO, ${ }^{1,2}$ Tomoaki KitamuRA, ${ }^{2}$ Kazushi HiguCHI, ${ }^{1}$ \\ and Kazuhiko NOZAKI ${ }^{2}$ \\ ${ }^{1}$ Department of Neurosurgery, Japanese Red Cross Nagahama Hospital, Nagahama, Shiga, Japan \\ ${ }^{2}$ Department of Neurosurgery, Shiga University of Medical Science, Otsu, Shiga, Japan
}

\begin{abstract}
We report a rare case of primary central nervous system (CNS) lymphoma as methotrexate-associated lymphoproliferative disorders (MTX-LPD). A 75-year-old woman who had been treated for rheumatoid arthritis (RA) with MTX for 3 years was admitted to our hospital complaining of unsteady gait, nausea, and vomiting. T2-weighted image of magnetic resonance imaging (MRI) showed multiple high intensity mass-like lesions including right lateral, frontal and temporal lobes, and right cerebellar hemisphere. We performed surgical biopsy, and the pathological and immunohistochemical examinations identified T-cell lymphoma. The tumor regressed and the symptoms were resolved soon after MTX withdrawal. Primary CNS lymphoma due to MTX-LPD is a rare disease and only eight cases including ours are reported.
\end{abstract}

Keywords: methotrexate-associated lymphoproliferative disorders, T-cell lymphoma, other iatrogenic immunodeficiency-associated lymphoproliferative disorders, rheumatoid arthritis, Epstein-Barr virus

\section{Introduction}

Methotrexate-associated lymphoproliferative disorders (MTX-LPD) ${ }^{1)}$ are one of other iatrogenic immunodeficiency-associated lymphoproliferative disorders (OIIA-LPD), defined in the classification of 4th edition of the World Health Organization (WHO). OIIA-LPD are lymphomas or lymphoid proliferations which develop in patients who are in immunodeficiency state by receiving immunosuppressive drugs (e.g., MTX) or immunomodulator drugs (e.g., anti-tumor necrosis factor $[\mathrm{TNF}]-\alpha){ }^{2}{ }^{2}$ The representative and in large part of OIIA-LPD is MTX-LPD from which the rheumatoid arthritis (RA) patients who take low-dose MTX weekly mainly suffer. MTX-LPD are still pathologically uncertain, although some reported features include high detection of Epstein-Barr Virus (EBV) in a immunohistochemistry study and high remission rate after cessation of MTX especially

Received July 10, 2020; Accepted September 11, 2020

Copyright $\subseteq 2021$ by The Japan Neurosurgical Society This work is licensed under a Creative Commons AttributionNonCommercial-NoDerivatives International License. among patients with EBV positive. ${ }^{3-5)}$ Most of the MTX-LPD first appears at nodal or extranodal sites on body trunk, with presenting symptoms of superficial lymphadenopathy, extranodal mass, so on. ${ }^{6)}$ A few primary CNS lymphomas in patients with MTX-LPD were reported. ${ }^{7-13)}$ We report a RA patient who developed primary CNS lymphoma as MTX-LPD.

\section{Case Report}

A 75-year-old woman was admitted to our hospital complaining of unsteady gait, nausea, and vomiting. She had medical history of RA which had been treated with MTX for 3 years, and anemia. She had also taken prednisolone 3 months before admission and Iguratimod, an immunosuppressant for RA 5 months before admission, besides MTX.

On admission, a physical examination showed no abnormal findings except body temperature was $37.7^{\circ}$. She was alert and had no headache and had no neurological symptom including facial or limb paresis, dysarthria, or aphasia. Laboratory findings were as follows: hemoglobin $8.2 \mathrm{mg} / \mathrm{dL}$, leukocyte count 9000 cells $/ \mu \mathrm{L}$, urea $11.2 \mathrm{mmol} / \mathrm{L}$, creatinine $0.79 \mathrm{mg} / \mathrm{dL}$, potassium $4.0 \mathrm{mmol} / \mathrm{L}$, sodium $139 \mathrm{mmol} / \mathrm{L}$, lactate 
dehydrogenase (LDH) 309 IU/L, and C-reactive protein $4.79 \mathrm{mg} / \mathrm{dL}$. Computed tomography (CT) scans of thoracoabdominal region showed no abnormalities. A head CT revealed multiple low-density lesions including right frontal, temporal and occipital lobes, and right cerebellar hemisphere and no contrast-enhanced lesions were detected (Fig. 1A). On brain magnetic resonance imaging (MRI), these lesions were hyperintense on T2-weighted imaging, fluid-attenuated inversion recovery and diffusion-weighted images (Figs. 1B and 1C). Most lesions demonstrated ring-like enhancement (Fig. 1D) and some parts showed open-ring enhancement (Fig. 1D, arrow) with gadolinium.

Although we suspected the brain lesions of metastatic tumors, an upper endoscopy and tumor marker tests (carcinoembryonic antigen [CEA], CA19-9 and $\alpha$-fetoprotein [AFP]) detected no malignant changes. A brain biopsy examination for a lesion of right frontal lobe was performed (Fig. 2A). Pathological examination showed a small round cell tumor around the vessels on hematoxylin and eosin staining (Fig. 2B), and immunohistochemical staining revealed that tumor cells were positive for CD3, CD4, CD5, CD8 and CD45(LCA) as T-cell markers (Fig. 2C-2G), and were negative for CD10, CD79a as B-cell markers (Fig. 2H and 2I), CD20, CD30, and CD56 as other markers (Fig. 2J-2L). In situ hybridization for EBV-encoded RNA (EBER) was negative (Fig. 2M). Results of additional laboratory analyses after the pathological examination were as follows: soluble interleukin-2 receptor $566 \mathrm{U} / \mathrm{mL}$, serum immunoglobulin $\mathrm{G}$ ( $\mathrm{IgG}$ ) to EBV virus capsid antigen (VCA) 320, serum IgM to EBV VCA <10, serum EBV-nuclear antigen (EBNA) 40.

A diagnosis of primary CNS T-cell lymphoma as OIIA/MTX-LPD was made by radiological and pathological findings above. The tumor regressed and the symptoms were resolved soon after MTX withdrawal (Fig. 3A). The patient started to take iguratimod again to control RA symptoms, and it did not affect tumor control. Duration of Cessation of iguratimod was 7 weeks. Complete remission has been obtained more than 9 months on MRI (Figs. 3B and 3C), and no recurrence of neurological symptom has occurred more than 20 months. Tumor regression after MTX cessation is known as distinguishable feature of MTX-LPD, and the clinical course of our patient was also consistent with the diagnosis of MTX-LPD.

\section{Discussion}

Head MRI as a screening examination for patient's complaints including unsteady gait, nausea, and vomiting revealed multiple cerebral lesions, and the results of the pathological and immunohistochemical examinations and medical history of using
MTX for RA treatment made a diagnosis of the disease as primary CNS T-cell lymphoma due to MTX-LPD.

The pathological types of reported primary CNS lymphoma as MTX-LPD were polymorphic/lymphoplasmacytic LPD, T-cell lymphoma, DLBCL, and intravascular large B-cell lymphoma. Concerning MTX-LPD of non-CNS sites, Hodgkin's lymphoma and natural killer/T-cell lymphoma were also reported. ${ }^{4-6)}$ The major type of MTX-LPD of non-CNS sites was DLBCL (53-65\%), and T-cell lymphoma was relatively rare $(3.0-10 \%)$.

Satou et al. ${ }^{7)}$ reported clinical characteristics of MTX-LPD of non-CNS sites based on 43 MTX-LPD patients: (1) median age was 66 years (range: $31-85$ ), (2) median duration of MTX using was 48 months (range: 6-252), (3) infiltration of small- to mediumsized lymphocytes in most cases of LPD which were immunohistochemically positive for CD3, CD4, but negative for CD8, (4) all CD8-positive T-cell LPD patients achieved complete remission after withdrawal of MTX regardless of EBV positivity, and (5) five of $43(12 \%)$ cases had EBV-positive tumor.

Seven cases of PCNSL derived from MTX-LPD were reported previously. ${ }^{8-14)}$ Representative characteristics of those seven cases with our case were as follows (Table 1): (A) median age was 58 years (range: 50-78), (B) primary illness were all RA, (C) median duration of MTX using was 48 months (range: 12-120), (D) three of seven (43\%) were DLBCL, two of seven (29\%) including our case were T-cell lymphoma, and one polymorphic/lymphoplasmacytic lymphoproliferative disorder case and one intravascular large B-cell lymphoma case were reported, respectively (each one is account for $14 \%$ ), (E) four of eight (50\%) cases had EBV-positive tumor, (F) tumor regression after cessation of MTX was obtained in six of seven ( $86 \%$ ) cases. Clinical characteristics of our case were same or belong to greater portion of these features of (A), (B), (C), (E), and $(\mathrm{F})$. Our case is unique in that T-cell, not B-cell, lymphoma due to MTX-LPD. EBV positivity of MTX-T-cell LPD is reported to be relatively rare as in our case, whereas that of MTX-B-cell LPD is relatively high. It is speculated that EBV positivity may be related to cell types not to site of disease.

Laboratory analyses of IgG to EBV VCA, IgM to EBV VCA and EBNA of our case showed infection of EBV, and that was common result worldwide. Katsuyama et al. ${ }^{5}$ showed that the positive result of peripheral blood EBV among MTX-LPD patients seem to be prognostic factor for better outcome. It is possible that good clinical course of our patient is associated with serum EBV-DNA positivity. 

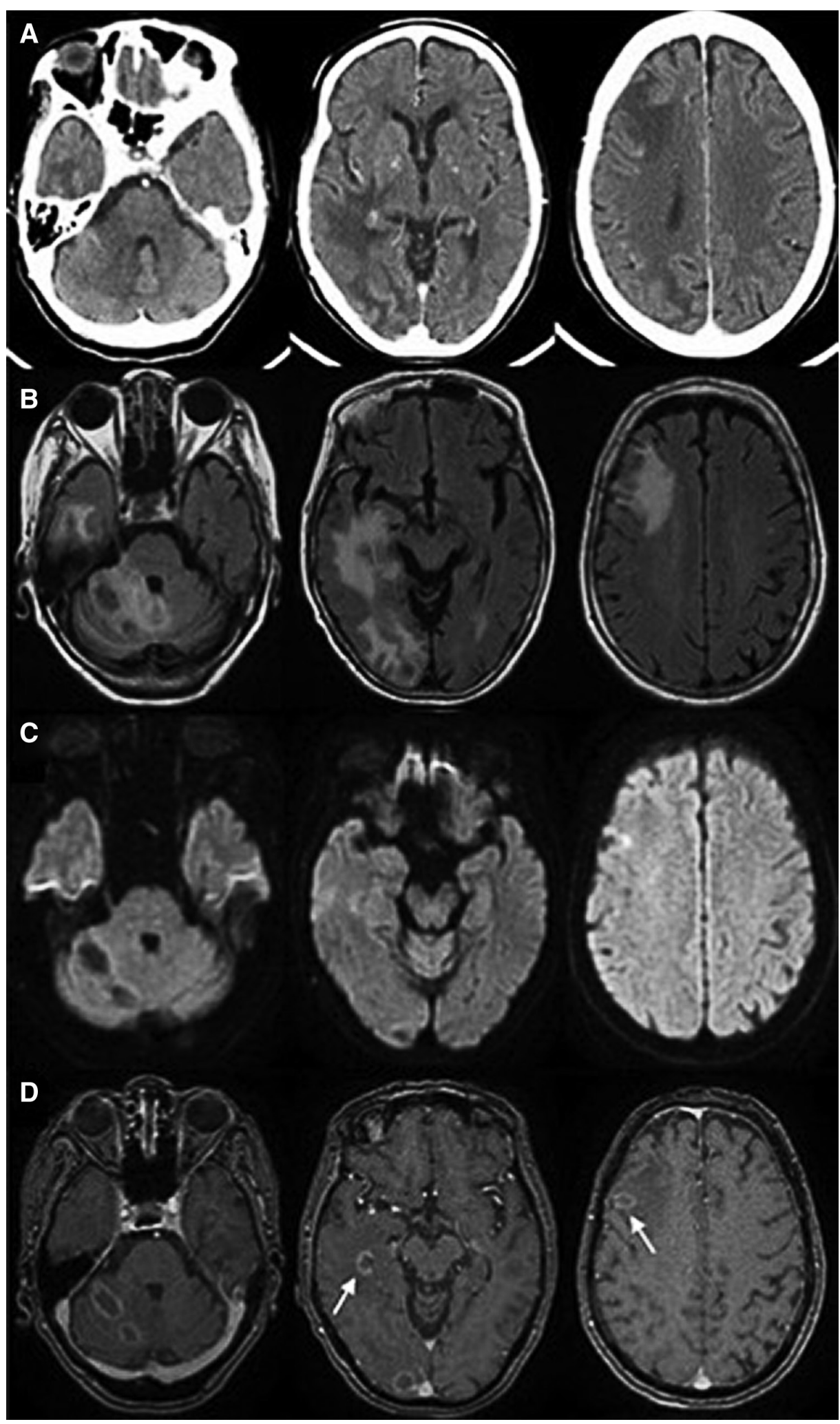

Fig. 1 CT and MRI images for screening test. (A) Contrasted CT showed multiple hypodensity lesions without contrast enhanced area. (B) FLAIR images demonstrating multiple hyperintensity lesions including right cerebellar and cerebral hemispheres. (C) Diffusion-weighted images demonstrated multiple hypointense lesions. (D) Gd-enhanced MRI images showed multiple ring-like enhanced lesions, and some lesions revealed open-ring sign (arrow). CT: computed tomography, FLAIR: fluid-attenuated inversion recovery, MRI: magnetic resonance imaging. 

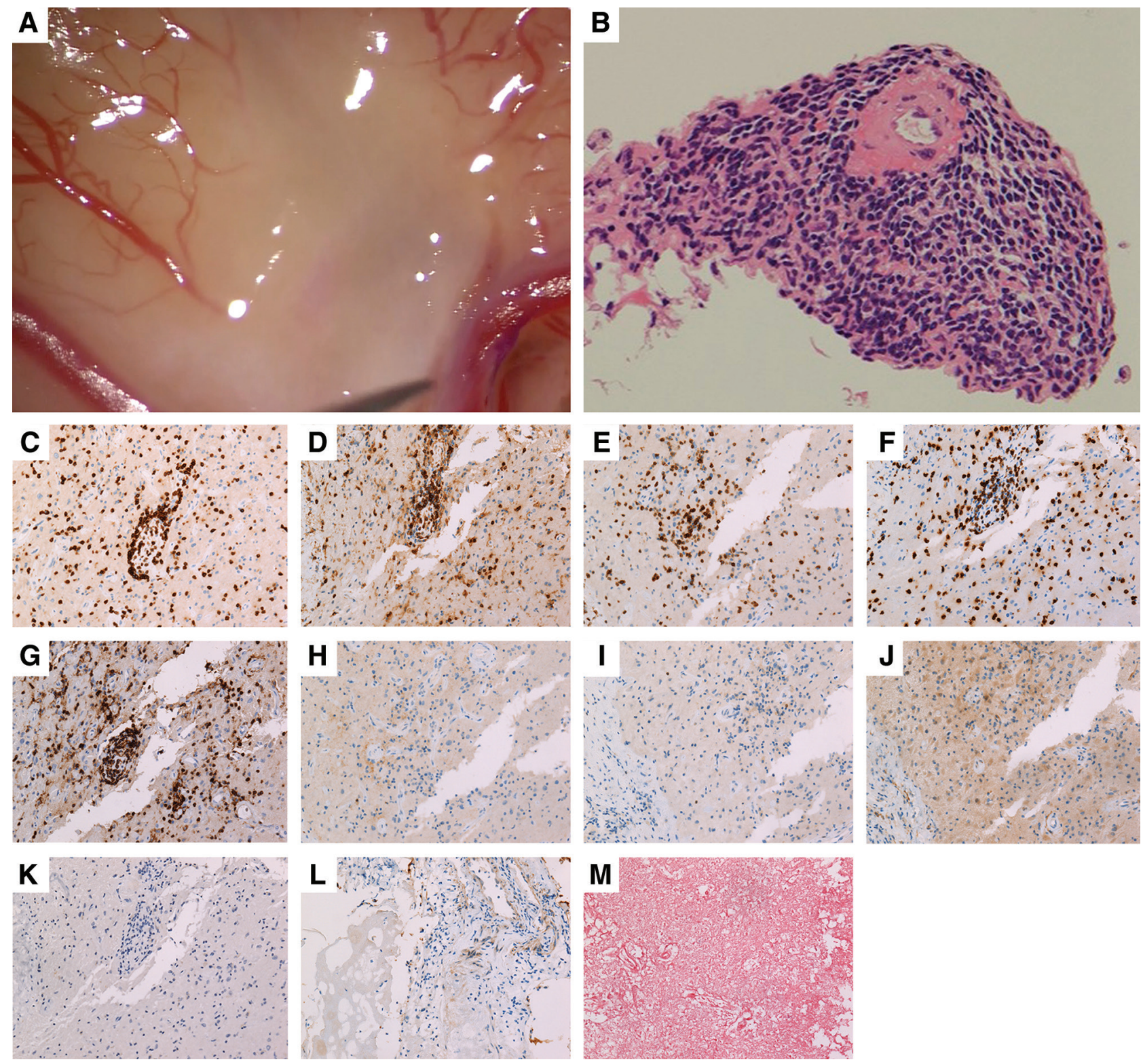

Fig. 2 (A) Biopsy; surface of right frontal lobe after removal of arachnoid membrane. A grayish white and jellylike soft tumor tissue was detected on cortical surface macroscopically. (B) Hematoxylin and eosin staining of a biopsy specimen from lesion of right frontal lobe showing small round cell tumor cells around the vessels. Immunohistochemical staining for CD3 (C), CD4 (D), CD5 (E), CD8 (F), CD45 (G) as T-cell markers were positive, and CD20 (H) CD79a (I) as B-cell markers, CD10 (J), CD30 (K), CD56 (L) as other markers were negative. (M) In situ hybridization for EBV encoded RNA was negative. EBV: Epstein-Barr virus.

Gd-enhanced MRI images of this case showed multiple ring-like enhanced lesions, and some lesions revealed open-ring sign. The former is found in $0-13 \%$ of non-acquired immunodeficiency syndrome primary central nervous system lymphoma (PCNSL) patients, ${ }^{15)}$ although the latter was reported specific findings of atypical brain demyelination. ${ }^{16)}$ Only two cases of open-ring enhancement in PCNSL patients were reported, ${ }^{17)}$ and no case was reported previously in PCNSL derived from MTX-LPD. ${ }^{8-14)}$ Usefulness of this finding for diagnosis of primary PCNSL as MTX-LPD needs to be confirmed in further study.

Idiopathic (not MTX-related) primary CNS T-cell lymphoma is also known as CNS T-cell LPD. Some features of the disease, mainly pathologically and immunohistochemically, ${ }^{18)}$ are similar to MTX-LPD of CNS sites, although clinical course of both diseases is thought to be different. Idiopathic primary CNS T-cell lymphoma needs treatment of steroid or chemotherapy including MTX with severe clinical 


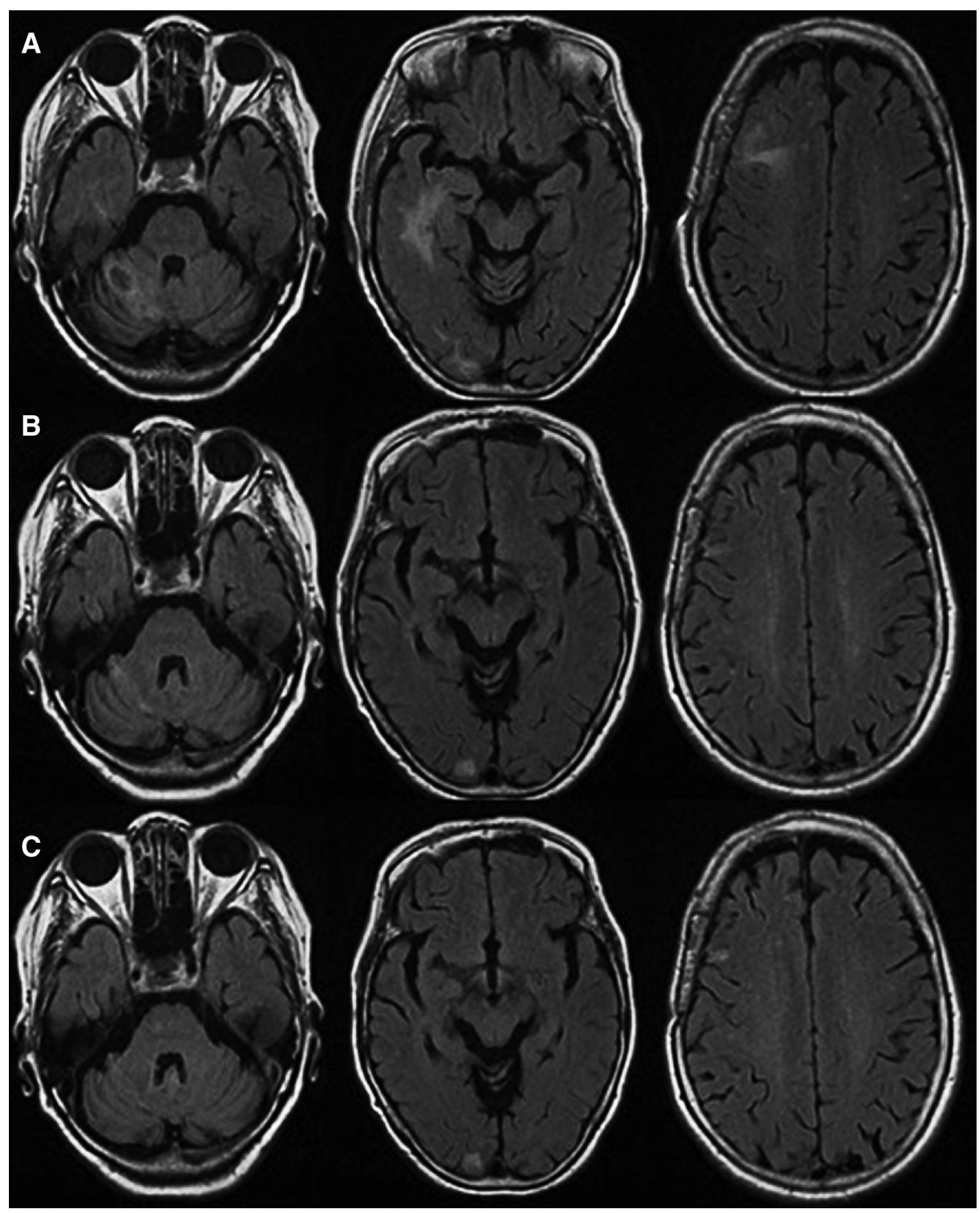

Fig. 3 Follow-up MRI (FLAIR): (A) 1 month, (B) 3 months, and (C) 9 months after MTX withdrawal. FLAIR: fluidattenuated inversion recovery, MRI: magnetic resonance imaging, MTX: methotrexate.

outcome in some cases. However, our case obtained long-term complete remission only by withdrawing MTX, the first case of primary CNS T-cell lymphoma as MTX-LPD $^{9)}$ needed high-dose MTX treatment, we need more cases to clarify features of the disease.

Our patient took Iguratimod, one of other immunomodulatory agents for treating RA, when she suffered from MTX-LPD. This drug is a kind of the disease-modified anti-rheumatic drug (DMARDs), inhibits B cells from producing immunoglobulins and autoantibodies, and downregulates T-cell-mediated cellular immunity. ${ }^{19)}$ MTX-LPD due to iguratimod had not been reported, although the influence of iguratimod to pathophysiology of MTX-LPD is still unclear.

One relapse case $^{9)}$ and one regrowth case ${ }^{14)}$ of PCNSL as MTX-LPD after withdrawal of MTX were reported; therefore, the rate of relapse/regrowth of PCNSL as MTX-LPD was $12.5 \%$ (two out of eight). 
Table 1 MTX-LPD cases in CNS

\begin{tabular}{|c|c|c|c|c|c|c|c|}
\hline & Age/sex & $\begin{array}{l}\text { Primary } \\
\text { illness }\end{array}$ & $\begin{array}{l}\text { MTX duration } \\
\text { (years) }\end{array}$ & Pathology & sIL-2R & EBER & $\begin{array}{l}\text { Regression } \\
\text { after cessation } \\
\text { of MTX }\end{array}$ \\
\hline Kleinschmidt ${ }^{8)}$ & $78 / \mathrm{F}$ & RA & 10 & P/L LPD & $\mathrm{U}$ & + & + \\
\hline Fukushima $^{9)}$ & $64 / F$ & RA & 4 & PTCL-NOS & 3210 & + & + transient \\
\hline Migita $^{10)}$ & $53 / \mathrm{F}$ & RA & 1 & DLBCL & 270 & + & + \\
\hline $\mathrm{Liu}^{11)}$ & $58 / \mathrm{M}$ & RA & 2 & DLBCL & $\mathrm{U}$ & - & $\mathrm{U}$ \\
\hline Shimada $^{12)}$ & $60 \mathrm{~s} / \mathrm{F}$ & RA & 7 & U & 391 & + & + \\
\hline Kikuchi $^{13)}$ & $50 / \mathrm{F}$ & RA & 3 & IVLBCL & 1373 & - & + \\
\hline Uchida $^{14)}$ & $52 / F$ & RA & 4 & DLBCL & 646 & - & - \\
\hline Present case & $70 \mathrm{~s} / \mathrm{F}$ & $\mathrm{RA}$ & 3 & $\begin{array}{c}\text { T-cell } \\
\text { lymphoma }\end{array}$ & 566 & - & + \\
\hline
\end{tabular}

Note: Modified Table 1 in reference 14 (Uchida Y et al, Rinsho Shinkeigaku 58: 485-491, 2018). DLBCL: diffuse large B-cell lymphoma, EBER: Epstein-Barr virus-encoded RNA, IVLBCL: intravascular large B-cell lymphoma, MTX: methotrexate, P/L LPD: polymorphic/lymphoplasmacytic lymphoproliferative disorder, PTCL-NOS: peripheral T-cell lymphoma, not otherwise specified, RA: rheumatoid arthritis, sIL-2R: soluble interleukin 2 receptor, U: unavailable.

Relapse/regrowth rate of MTX-LPD of non-CNS sites after cessation of immune suppressive drugs (ISDs) including MTX was reported $13.5 \%{ }^{20)}$ Iguratimod is classified into DMARDs which suppressive effect on lymphocyte proliferation is weak compared to ISDs (MTX, cyclophosphamide, cyclosporine, etc.). Development of MTX-LPD is thought to be caused by ISDs which accelerate the impairment of anti-LPD immunity, ${ }^{20)}$ therefore influence of iguratimod to MTX-LPD recurrence is expected lower than that of ISDs. Although iguratimod is a relatively new drug for treating RA approved in 2012, it is unclear whether long-term administration of this drug may affect relapse/regrowth of PSNCL as MTX-LPD. Our patient has continued iguratimod more than 18 months after 7 weeks cessation for MTX-LPD, and no relapse of neurological symptom has occurred within the period.

\section{Conclusion}

We reported a rare case of primary CNS T-cell lymphoma as OIIA/MTX-LPD. Withdrawal of MTX is able to obtain regression of disease on some MTX-LPD cases as our patient; thus, it is highly important to diagnose the disease as soon as possible.

\section{Conflicts of Interest Disclosure}

This research did not receive any specific grant from funding agencies in the public, commercial, or not-for-profit sectors. None of the authors have no conflicts of interest. All authors have registered online Self-reported COI Disclosure Statement Forms through the website for JNS members.

\section{References}

1) Ellman MH, Hurwitz H, Thomas C, Kozloff M: Lymphoma developing in a patient with rheumatoid arthritis taking low dose weekly methotrexate. $J$ Rheumatol 18: 1741-1743, 1991

2) Hasserjian RP, Chen S, Perkins SL, et al.: Immunomodulator agent-related lymphoproliferative disorders. Mod Pathol 22: 1532-1540, 2009

3) Salloum E, Cooper DL, Howe G, et al.: Spontaneous regression of lymphoproliferative disorders in patients treated with methotrexate for rheumatoid arthritis and other rheumatic diseases. J Clin Oncol 14: 1943-1949, 1996

4) Ichikawa A, Arakawa F, Kiyasu J, et al.: Methotrexate/ iatrogenic lymphoproliferative disorders in rheumatoid arthritis: histology, Epstein-Barr virus, and clonality are important predictors of disease progression and regression. Eur J Haematol 91: 20-28, 2013

5) Katsuyama T, Sada KE, Yan M, et al.: Prognostic factors of methotrexate-associated lymphoproliferative disorders associated with rheumatoid arthritis and plausible application of biological agents. Mod Rheumatol 27: 773-777, 2017

6) Hoshida Y, Xu JX, Fujita S, et al.: Lymphoproliferative disorders in rheumatoid arthritis: clinicopathological analysis of 76 cases in relation to methotrexate medication. J Rheumatol 34: 322-331, 2007

7) Satou A, Tabata T, Miyoshi H, et al.: Methotrexateassociated lymphoproliferative disorders of T-cell phenotype: clinicopathological analysis of 28 cases. Mod Pathol 32: 1135-1146, 2019

8) Kleinschmidt-DeMasters BK, Damek DM, Lillehei KO, Dogan A, Giannini C: Epstein Barr virus-associated primary CNS lymphomas in elderly patients on immunosuppressive medications. J Neuropathol Exp Neurol 67: 1103-1111, 2008 
9) Fukushima M, Katayama Y, Yokose N, et al.: Primary central nervous system malignant lymphoma in a patient with rheumatoid arthritis receiving low-dose methotrexate treatment. Br J Neurosurg 27: 824-826, 2013

10) Migita K, Miyashita T, Mijin T, et al.: Epstein-Barr virus and methotrexate-related CNS lymphoma in a patient with rheumatoid arthritis. Mod Rheumatol 23: 832-836, 2013

11) Liu W, Xue J, Yu S, Chen Q, Li X, Yu R: Primary central nervous system lymphoma mimicking recurrent depressive disorder: a case report. Oncol Lett 9: 1819-1821, 2015

12) Shimada H, Dobashi H, Morimoto H, et al.: Primary central nervous system lymphoma in a rheumatoid arthritis patient treated with methotrexate: a case report. BMC Res Notes 88: 1-7, 2015

13) Kikuchi J, Kaneko Y, Kasahara H, et al.: Methotrexateassociated intravascular large B-cell lymphoma in a patient with rheumatoid arthritis. Intern Med 55: 1661-1665, 2016

14) Uchida Y, Hokkoku K, Hatanaka Y, Kikuchi Y, Tashiro H, Sonoo M: Primary central nervous system methotrexate associated lymphoproliferative disorders in a patient with rheumatoid arthritis. Rinsho Shinkeigaku 58: 485-491, 2018

15) Haldorsen IS, Espeland A, Larsson EM: Central nervous system lymphoma: characteristic findings on traditional and advanced imaging. AJNR Am J Neuroradiol 32: 984-992, 2011

16) Masdeu JC, Quinto C, Olivera C, Tenner M, Leslie D, Visintainer P: Open-ring imaging sign: highly specific for atypical brain demyelination. Neurology 54: 14271433, 2000

17) Zhang $\mathrm{D}, \mathrm{Hu}$ LB, Henning TD, et al.: MRI findings of primary CNS lymphoma in 26 immunocompetent patients. Korean J Radiol 11: 269-277, 2010

18) Menon MP, Nicolae A, Meeker H, et al.: Primary CNS T-cell lymphomas: a clinical, morphologic, immunophenotypic, and molecular analysis. Am J Surg Pathol 39: 1719-1729, 2015

19) Jiang H, Gao H, Wang Q, Wang M, Wu B: Molecular mechanisms and clinical application of Iguratimod: a review. Biomed Pharmacother 122: 109704, 2020

20) Tokuhira M, Tamaru JI, Kizaki M: Clinical management for other iatrogenic immunodeficiency-associated lymphoproliferative disorders. J Clin Exp Hematop 59: 72-92, 2019

Corresponding author: Hiroto Kawano, MD Department of Neurosurgery, Shiga University of Medical Science Hospital, 6 Setatsukinowacho, Otsu, Shiga 520-2192, Japan. e-mail: hkawano@belle.shiga-med.ac.jp 